\title{
¿Para qué sirve la formación en prevención de riesgos laborales? Reflexiones teóricas e implicaciones prácticas a partir del caso de los empleos descualificados en subcontratistas intensivos que actúan como eslabón final de la cadena de subcontratación en el sector de la construcción en España
}

\author{
David HERNÁNDEZ SALGUERO \\ Universitat Autònoma de Barcelona \\ David.hernandezs@e-campus.uab.cat
}

Recibido: 15-07-2013

Aceptado: 24-05-2014

\begin{abstract}
RESUMEN
En este trabajo se analiza la utilidad de la formación preventiva impartida a una muestra de trabajadores descualificados empleados en subcontratistas intensivos en el sector de la construcción. Durante el periodo de análisis son regulados nuevos cursos de formación desde el convenio colectivo creando un marco dual de formaciones, legal y convencional, que afrontan idénticos problemas de calidad. ¿Hasta qué punto el modelo de formación puede permitir alcanzar los objetivos de reducir la siniestralidad? Datos de una investigación empírica nos permite concluir que la formación impartida ha servido más para cumplir con un objetivo credencialista que como herramienta de reducción de los accidentes de trabajo.
\end{abstract}

Palabras clave: formación preventiva, accidentes de trabajo, inmigración, construcción.

What for is used preventive training? Theoretical discussion and practical implications: The case of unqualified jobs in the subcontracting firms in the construction sector in Spain

\begin{abstract}
This work analyses the usefulness of preventive training, which has been delivered to a sample of unqualified workers in subcontracting firms. Within this period of analysis new courses are designed by social partners, which define a framework of training regulated by law and collective bargaining. Both types face similar problems of quality with regards to features of the sector and employers. In this scenario should be considered the effectiveness of training in achieving the goal of reducing of accidents. Empirical research provides with the conclusion that this training has been used as a mean to obtained a diploma and to achieve a credentialist objective rather than as a tool for reducing work-related accidents.
\end{abstract}

Keywords: preventive training, work-related accidents, migrant labour, construction’ sector. 


\section{REFERENCIA NORMALIZADA:}

Hernández Salguero, D. (2015) “¿Para qué sirve la formación en prevención de riesgos laborales? Reflexiones teóricas e implicaciones prácticas a partir del caso de los empleos descualificados en subcontratistas intensivos que actúan como eslabón final de la cadena de subcontratación en el sector de la construcción en España”. Cuadernos de Relaciones Laborales, Vol. 33, núm.2, p. 331-356.

SUMARIO: Introducción. 1. La formación en prevención de riesgos laborales. 2. Teorías de la formación. 3. Descripción de los estudios de caso. 4. Análisis crítico del modelo vigente. 5. Conclusión. 6. Notas finales. 7. Bibliografía.

\section{Introducción}

La formación es una actividad clave e indispensable para la prevención de riesgos laborales, tanto para las empresas como para los trabajadores. Para las empresas representa una medida que puede contribuir a reducir los accidentes y mejorar la productividad (Bena, et al 2009; Gondelhar et al, 2001) ${ }^{1}$, mientras que para los trabajadores es un medio de conocimiento de su entorno laboral, de los riesgos a los que pueden estar expuestos y de cómo prevenirlos de manera efectiva.

La legislación española y europea establece la obligación de formar en materia preventiva y el derecho de los trabajadores a ser formados e informados sobre los riesgos que tienen en su puesto de trabajo y acerca de cómo evitarlos. Es necesaria en la construcción de un conjunto de competencias clave para el ejercicio de la profesión conforme a las exigencias de su trabajo, que necesariamente debe englobar la cualificación profesional de los trabajadores ${ }^{2}$, en todo caso, siguiendo una tendencia de modernización y mejora, que se corresponde con las políticas de similar signo emprendidas en otros países de la Unión Europea.

La formación debe considerarse entonces, una medida de prevención y la falta de formación una de las posibles causas a considerar en la explicación de los accidentes y daños a la salud de los trabajadores. Es por ello que en sectores con mucha siniestralidad se pone el acento en su realización e incluso se diseñan programas específicos para los trabajadores ocupados en ciertas tareas. Este es el caso del sector de la construcción en España.

El IV convenio del sector de la Construcción 2007-2011 estableció la obligatoriedad de diversos tipos de programas de formación y creó los mecanismos para su control y gestión.

Dichos programas son considerados por los agentes sociales como adecuados para cumplir con las obligaciones de formación que resultan de las regulaciones legales ${ }^{3}$. Además, los Servicios de Prevención concertados por cada empresa siguen teniendo la función de impartir una formación a medida de las necesidades de cada situación laboral. No obstante, diversos factores característicos del sector, como la enorme flexibilidad organizativa de las empresas constructoras, han influido enormemente en la calidad de la formación y en las posibilidades de impartirla.

En este artículo se analiza la llamada 'formación preventiva' impartida a un reducido grupo de empresas subcontratistas en el sector de la construcción en España, interpretándola a la luz de diferentes planteamientos teóricos sobre el papel 
de la formación, como la Teoría del Capital Humano y la Teoría Credencialista. En este sentido, es un intento de demostración empírica de la actual validez de los planteamientos de Collins.

Los resultados indican que la formación preventiva ha sido utilizada como un medio para cumplir formalmente con los requisitos legales y poder entrar en las obras a trabajar, más que como una verdadera medida de prevención de accidentes. Estos resultados se refieren sólo a una muestra de empresas inferiores en la cadena de subcontratación en el sector de la construcción. Resolver esta hipótesis en un entorno mayor de empresas requiere un estudio más amplio.

\section{La formación en prevención de riesgos laborales}

Las competencias y cualificaciones en materia de prevención de riesgos laborales forman un universo de conocimientos especializados bastante complejo, con diferentes tipos de programas formativos con objetivos muy dispares, abarcando desde la formación preventiva de nivel medio o superior requerida para acceder al ejercicio de la profesión de 'técnico de prevención de riesgos laborales', hasta la formación que tiene por objeto dotar a los trabajadores de los conocimientos necesarios para "trabajar" de manera segura. En el presente trabajo nos centraremos en este último tipo de formación preventiva.

La legislación actual en España sobre este tema distingue entre la 'formación específica' y la 'formación genérica' (Ley 31/95 de prevención de riesgos laborales y RD 39/97 del reglamento de los servicios de prevención.). En este sentido, toda la formación regulada para el ejercicio de la profesión de técnico en prevención de riesgos laborales (medio o superior), así como la de técnico de nivel básico se considera una formación genérica dentro del ámbito de la prevención.

La legislación establece también que los trabajadores que ejercen funciones productivas deben poseer el nivel de competencia y de conocimientos necesarios para su realización en condiciones seguras, lo cual significa que deben recibir una formación específica para poder prevenir los riesgos a los que están expuestos. Desde este punto de vista, la mayor parte de la formación que se puede recibir en un trabajo tenderá a ser específica, es decir, adaptada a los riesgos del puesto de trabajo concreto de que se trate. Se trata de una formación "en el empleo", no transferible a otros puestos de trabajo ni a otras empresas. El propósito de esta formación recoge los principios y objetivos del artículo 19 de la LPRL y de los Reglamentos que la desarrollan. Su realización suele estar planificada a lo largo del año por el Servicio de Prevención (propio o ajeno) y es impartida por técnicos de prevención de nivel superior pertenecientes a dicho Servicio de Prevención.

El término "específico" hace referencia a un conjunto de riesgos o a una actividad concreta de las que puede llegar a realizar un trabajador en el desarrollo de su puesto de trabajo. Tales casos tienen que ver con los trabajos en altura, en espacios confinados, en exposición a determinados contaminantes como el amianto o en el montaje de plataformas de trabajo, por poner algunos ejemplos. La 
cualificación adecuada para realizar estos trabajos de manera segura implica la realización de una formación específica y la adquisición de una serie de competencias por parte del trabajador.

Ciertos tipos de conocimientos necesarios para la realización segura del trabajo, como por ejemplo los vinculados al manejo de un equipo de trabajo o de una máquina, pueden ser adquiridos a través de empresas especializadas que cuenten con los medios adecuados para su realización. En estos casos, aunque se trate de una formación específica, que puede ser considerada también como una formación "para el empleo", proporciona al trabajador una credencial, una señal de los conocimientos y habilidades adquiridos, que pueden ser transferibles a otras empresas y mejorar su posición en el mercado laboral. Cuando el trabajador se marcha, se lleva consigo dicha credencial.

Por lo tanto, además de atender al grado de transferibilidad de dicha formación al mercado, hay que valorar los aspectos relativos a la utilidad de sus contenidos para la prevención de riesgos (de acuerdo con las exigencias de la LPRL).

Por otro lado, el IV Convenio General del Sector para los años 2007-2011, establece la obligatoriedad de ciertos programas o cursos de formación en prevención de riesgos laborales, todavía hoy vigente según el V convenio del sector.

Estos cursos se clasifican en dos ciclos. El primer ciclo consta de un solo curso de 8 horas de duración, con un contenido muy genérico y una amplia diversidad de temas (véase la tabla 1). Ello facilita que en el aula puedan concurrir trabajadores de diversos puestos de trabajo y de oficios diferentes al mismo tiempo.

Este curso se define como la acción formativa inicial mínima en materia de prevención de riesgos laborales, cuyo objetivo principal es conseguir que los trabajadores adquieran los conocimientos necesarios para identificar tanto los riesgos laborales más frecuentes, como las medidas preventivas a implantar.

Sólo es teórico y no se establecen ejercicios prácticos en el convenio, aunque un formador, motu proprio, podría dinamizar la clase con algún ejercicio sencillo. 
Tabla 1. Contenido del curso inicial de $8 \mathrm{~h}$.

A) Conceptos básicos sobre seguridad y salud.

- El trabajo y la salud. Los riesgos profesionales. Factores de riesgo.

- Marco normativo básico en materia de prevención de riesgos laborales.

Deberes y obligaciones básicas en esta materia.

B) Técnicas preventivas elementales sobre riesgos genéricos.

- Caídas a distinto nivel, manipulación de cargas, medios de protección colectiva, equipos de protección individual, etc.

- Medios auxiliares (andamios colgados, modulares, borriquetas, etc.) • Equipos de trabajo (aparatos elevadores, pequeña maquinaria, etc.). • Señalización. • Simbología de los productos y materiales utilizados en las obras de construcción.

C) Primeros auxilios y medidas de emergencia.

- Procedimientos generales. • Plan de actuación.

D) Derechos y obligaciones. • Participación, información, consulta y propuestas.

Fuente: V convenio colectivo de la construcción, artículo 143 en www.trabajoenconstruccion.com/resources/subirarchivos/00071440archivo.pdf

El segundo ciclo consta de varios cursos y se configura en función del puesto de trabajo o familia profesional. Tiene un duración de 20 horas. (véase tabla 2). 


\section{Tabla 2. Contenido curso de segundo ciclo para albañiles}

\section{A. Definición de los trabajos.}

- Fachadas (fábrica de ladrillo y revestimiento de cemento). • Distribución interior. • Materiales (cerámicos, cartón-yeso, escayola, etc.).

B. Técnicas preventivas específicas.

- Aplicación del plan de seguridad y salud en la tarea concreta. Evaluación de riesgos en el caso de que no exista plan.

- Protecciones colectivas (colocación, usos y obligaciones y mantenimiento). • Protecciones individuales (colocación, usos y obligaciones y mantenimiento).

C. Medios auxiliares, equipos y herramientas.

- Andamios. - Borriquetas. - Plataformas de trabajo, pasarelas, etc.

D. Verificación, identificación y vigilancia del lugar de trabajo y su entorno.

- Riesgos y medidas preventivas necesarias.

- Conocimiento del entorno del lugar de trabajo. Planificación de las tareas desde un punto de vista preventivo.

- Manipulación de productos químicos. Ficha de datos de seguridad. Simbología.

E. Interferencias entre actividades. • Actividades simultáneas o sucesivas.

F. Derechos y obligaciones.

- Marco normativo general y específico. • Organización de la prevención. • Fomento de la toma de conciencia sobre la importancia de involucrarse en la prevención de riesgos laborales. • Participación, información, consulta y propuestas. • Duración del módulo: veinte horas.

Fuente: V Convenio de la Construcción, artículo 150 en

www.trabajoenconstruccion.com/resources/subirarchivos/00071440archivo.pdf.

La credencial que se expide con cualquiera de estos dos ciclos pertenece al trabajador y puede hacerla valer ante cualquier empresa del sector. Desde el punto de vista del mercado, se trata de una formación general. En cuanto a su contenido, pese a la especialización de las materias en el sector de la construcción, su tratamiento es superficial y general y no implica una formación estrictamente específica, pues no logra satisfacer las necesidades inmediatas de los trabajadores asistentes, por lo menos de los trabajadores observados en la investigación. De ello dan cuenta algunos testimonios recogidos en el aula durante la exploración:

(...) me aburro hasta yo misma dando esta clase, además están hartos de escuchar siempre lo mismo, eso si me entienden, (...) hoy tengo pladuristas (sic) que no trabajan nunca en andamios de fachada, más los tres marroquíes que ponen bordillos, pero claro, esto está en el programa (...)Se trata de una técnica formadora especializada en el sector durante una sesión del módulo específico de seis horas en el que asisten tres trabajadores de uno de los casos de estudio (caso B).

Sin embargo, hay que advertir que el segundo ciclo está pensado para ser una formación directamente relacionada con el puesto de trabajo, lo cual le da cierto 
grado de especificidad. Esta formación está a medio camino entre lo que sería una formación "para el empleo" y una formación "en el empleo"5.

Tras la entrada en vigor de la obligatoriedad de estos programas en 2008, las empresas que empleaban a trabajadores sin esta formación tuvieron que procurar que sus trabajadores realizaran el primer ciclo de ocho horas, puesto que sin ella no podían continuar trabajando. Esta formación se realizó generalmente por encargo de sus empresas, y en muchos casos dentro del horario de trabajo. Los trabajadores sin esta formación se convirtieron en un recurso no utilizable en este contexto.

En teoría, mediante esta formación se pretendió satisfacer las obligaciones derivadas del artículo 19 de la LPRL, pero en la práctica ello parece dudoso. A pesar de que pudiera ser interpretado de esta manera, se trata de un debate abierto en el que instituciones como la Inspección de Trabajo o el Departament de Treball de la Generalitat de Catalunya se han pronunciado en sentido adverso. ${ }^{6}$ Como se verá más adelante, muchas de las necesidades detectadas en las visitas de obra responden a un grado de especificidad no resuelto en la formación.

Hasta finales de 2008 la única formación exigida en las obras era la que impartían los Servicios de Prevención de cada empresa. Esta formación tenía una duración de entre una y dos horas. A partir de la generalización de la formación derivada del Convenio conocida como aula permanente o curso de 8h, imprescindible para poder trabajar en la construcción, las empresas observadas no han facilitado la realización de la formación del servicio de prevención.

\section{Teorías de la formación}

La formación en prevención de riesgos laborales es a menudo objeto de discusión por parte de profesionales y empresarios ${ }^{7}$. En el debate siempre se cuestiona el grado de eficacia de esta actividad. Aunque con frecuencia no existe acuerdo sobre cómo definir la eficacia de la formación, algunos autores (Bena et al, 2009 y Becker y Morawetz, 2004) apuntan dos aspectos clave de la calidad del proceso formativo que en este trabajo hemos demoninado la transferencia de su contenido al medio laboral y el retorno. El primer concepto indica cuánto se utiliza en el trabajo de lo aprendido en la actividad formativa y cuánto tiempo perdura. El segundo concepto es expresión de la eficacia de la formación en términos económicos.

En el fondo estos dos aspectos forman parte de un largo debate académico sobre qué es y para qué sirve la formación, y que ha tenido como protagonistas a teorías tan relevantes como la Teoría del capital humano (Becker, 1993) o la Teoría credencialista (Collins, 1989). Estas dos teorías, sin menoscabo de otras como la Teoría de la reproducción (vease p.e. Bourdier y Passeron, 1969.) o la teoría neomarxista (Bowles y Gintis, 1985), pueden ser apropiadas para el análisis de la formación preventiva. 
Desde nuestro punto de vista, la Teoría del capital humano aporta tres elementos interesantes al debate de la formación preventiva.

- Por un lado diferencia entre 'formación específica' y 'formación genérica'. La especificidad, como ya se ha dicho, es una de las características importantes de este tipo de formación, ya que tiene que ver con el lugar de trabajo y con los riesgos concretos a los que está expuesto el trabajador.

- Además, la especificidad está ligada a la cuestión de la inseparabilidad entre la cualificación y el trabajador, de manera que el abandono de un trabajador supone la pérdida de las competencias de ese trabajador y de sus credenciales por parte de la empresa. En ello radica precisamente la diferencia entre el capital humano y el capital financiero (Becker, 1993).

- Finalmente, esta teoría plantea el debate sobre los efectos de la formación en la productividad. La formación profesional general suministra competencias y capacitaciones genéricas, que por tanto pueden ser de utilidad para muchas empresas de sectores de actividad diferentes (Alcaide, González y Flores, 1996). ${ }^{8}$ Así, las empresas que promueven la formación en el trabajo incrementarán (presumiblemente) la productividad marginal futura de sus trabajadores. Pero si se trata de una formación general, también pueden incrementar la productividad marginal de otras empresas (Becker, 1993:33) que en el futuro puedan contratar a estos trabajadores.

Desde esta perspectiva, la formación completamente 'específica' puede ser definida como aquella formación que sólo es útil para el puesto de trabajo de la empresa en cuestión, pero no tiene ningún efecto en la productividad del trabajador si éste va a otra empresa. Asimismo, los costes de contratación, honorarios de las agencias, tiempo empleado en las entrevistas y en la comprobación de las referencias etc. son también formas específicas de capital humano, aunque no sean formación. Forman parte del capital humano porque pierden su valor cuando el trabajador se marcha. Si toda la formación fuera completamente específica, el salario que un empleado pudiera conseguir en cualquier otro lugar sería independiente de la cantidad de formación que hubiera recibido (Becker, 1993:41).

La visión en términos economicistas o de productividad que se desprende de la Teoría del capital humano choca con la visión propuesta desde la Teoría credencialista. Esta teoría, de tradición neoweberiana, permite poner en duda los efectos de la formación en la productividad, sin negarlos totalmente, y pone el énfasis en el valor de los diplomas para la sociedad. Según Collins (1989) la educación y la formación no están asociadas a la productividad de los empleados a un nivel individual, y las técnicas en el trabajo se aprenden mediante oportunidades que permitan su práctica. Las credenciales formativas son objeto de un especial énfasis en las empresas que dan una gran importancia al control normativo, pero no garantizan el éxito profesional.

Las enseñanzas superiores y profesionales servirían al propósito de elevar el 
status de ciertas profesiones y de establecer barreras entre practicantes y extraños, porque en realidad, muchas de las técnicas utilizadas en los empleos cualificados se aprenden en el mismo lugar de trabajo. De este modo Collins viene a decir que el aprendizaje descansa a menudo más en la experiencia en el propio trabajo que en la enseñanza formal de las escuelas.

De acuerdo con este planteamiento, las diferentes habilidades (innatas o adquiridas) que determinan el éxito en la escuela, están ligadas sólo de forma relativa al posterior éxito ocupacional, ya que están medidas a través del valor de certificación de las cualificaciones académicas (Collins, 1989:39). En resumen, el diploma prevalece sobre la propia enseñanza.

Este autor observa que durante los años setenta (del pasado siglo) los ingresos de los graduados cayeron, lo cual condujo a muchos economistas a preocuparse por el problema de la 'sobreeducación'. En este contexto, el concepto de 'capital humano' en sí mismo es puesto en duda, como reconoce el propio Becker (1993:17). Para Collins:

Con una terminación de estudios superiores casi universal y la mitad de los cohortes juveniles asistiendo a las facultades, estos valiosos objetivos pierden mucho de su demanda (...). La razón de que la educación sea parte de un sistema de estratificación cultural y la razón de que muchos estudiantes estén en las escuelas es que ellos necesitan un buen empleo. Esto significa que las razones para ir a los centros de enseñanza son ajenas a cualquier cosa que pueda suceder en las aulas (Collins, 1993:215).

Lo más interesante de esta idea es que hoy día puede ocurrir algo parecido con estudios que están muy lejos de tener la importancia social de un título universitario. Desde nuestro punto de vista, existe cierta conexión entre esta idea y lo que está ocurriendo con la 'formación preventiva' en el sector de la construcción. Los trabajadores pasan por el aula de formación con poca convicción, porque necesitan un empleo o porque así lo ordena su empresa. Un trabajador armenio comenta en una entrevista: No recuerdo cuando lo hice pero era de seguridad (...) era lo mismo que todos, nos enseñan lo que sabemos, es sólo teoría, lo práctico ya lo sabes (...) el primero fue aquí, un sábado, no se cuánto tiempo y otro de 24 horas creo, muy general.

El paso por el aula beneficia a la empresa, porque adquiere credibilidad ante sus clientes y ante la autoridad laboral. Pero ni los trabajadores ni los empleadores necesitan estar demasiado interesados en lo que pasa en el aula, y menos aún con aquello que pueda interferir con los modos de producción (rutinas, tareas) aceptado por unos y otros. El siguiente testimonio aclara en este sentido:

Compiten con otras empresas. Van reventados de tiempo y de dinero. (...) y nosotros eso no lo controlamos, les dejamos hacer siempre y cuando lo hagan bajo nuestro criterio (...). Testimonio de un encargado de contratista entrevistado en una obra de Sant Cugat en visita a empleador del caso C. 
A partir de estas ideas, a continuación se hará un análisis crítico de la formación preventiva basado en el análisis de tres estudios de caso del sector de la construcción

\section{Descripción de los estudios de caso}

Después de un largo periodo de observación iniciado en 2005 en numerosas obras del área metropolitana de Barcelona, son escogidas tres empresas del sector como casos de estudio. El estudio finaliza en 2011 tras un año y medio dedicado a analizar la información y realizar las entrevistas a los trabajadores. Se trata de dos empresas con empleadores extranjeros, denominados casos B y C, y una tercera con empleador español, denominado caso A. Las tres empresas tienen en común su posición y función en el sector y el tipo de trabajador contratado.

Se trata de subcontratistas intensivos que actúan como eslabón final de la cadena de subcontratación, directamente dependientes de la contratista. Se consideran eslabón final porque solo aportan mano de obra y por lo tanto no pueden subcontratar, de acuerdo con lo contenido en el artículo 5.f. de la ley de subcontratación. Su función principal, según se ha observado, es dotar de flexibilidad al proceso productivo, tanto de manera funcional en el desarrollo de diversas tareas en diversos momentos de la obra, como numérica aportando el número de trabajadores necesarios en cada momento.

El subcontratista intensivo es una categoría muy interesante de subcontratista de cuya importancia conocemos a través de sus protagonistas, como destacan los representantes sindicales. Véase los testimonios siguientes:

Estas empresas están ahí nada más que para aprotar trabajo (...) hoy dame 30, mañana sólo necesito $10(\ldots)$, estos son los que llamamos pistoleros. Entrevista a cargo sindical, especializado en el sector en el Vallès Occidental, realizada en 2005.

(...) y además hay especialización, se ven muchas empresas de "bordilleros" marroquíes, y de "tabiqueros" pakistaníes, (...) muchos extranjeros al final acaban trabajando para prestamistas de las grandes (...) eso ha sido muy fuerte durante estos años. Entrevista a técnico sindical de formación especializado en el sector, realizada en Barcelona en 2010.

Desafortunadamente el REA o registro de empresas acreditadas del sector no distingue entre categorías de subcontratistas y contratistas, menos aún por tipo de subcontratista, de cuyo rastro sólo queda constancia en los libros de subcontratación de cada obra. Su elección responde a una doble justificación: por un lado su idoneidad para el objeto de estudio -relación entre formación, inmigración y siniestralidad- y por otro lado, por motivos de accesibilidad a la realidad objeto de estudio, pues el observador es técnico en prevención y puede mantener un contacto regular con los casos de estudio. Véase la siguiente tabla para una muestra de datos esenciales correspondientes a los años 2008-9: 
Tabla 3. Resumen descriptivo. Muestra para año 2008-9

\begin{tabular}{|c|c|c|c|c|c|}
\hline Caso & $\begin{array}{l}\text { Plantilla } \\
\text { media }^{(a)}\end{array}$ & $\begin{array}{l}\text { Tipos de } \\
\text { trabajo }\end{array}$ & $\begin{array}{l}\text { Ïndice de } \\
\text { siniestrali- } \\
\text { dad }^{(\mathrm{c})}\end{array}$ & $\begin{array}{l}\text { Extranjeros } \\
\text { en plantilla } \\
\%\end{array}$ & $\begin{array}{l}\text { Tempo- } \\
\text { rali- } \\
\text { dad. \% }\end{array}$ \\
\hline B & 81 y 55 & $\begin{array}{l}\text { Vialidad y } \\
\text { obra civil }\end{array}$ & $18.44(7.56)$ & $100 \%$ & $88.9 \%$ \\
\hline $\mathrm{C}$ & 41 y 48 & $\begin{array}{l}\text { Encofrado y } \\
\text { tareas de } \\
\text { asistencia }\end{array}$ & $12.22(1.34)$ & $95 \%$ & $64.2 \%$ \\
\hline \multirow[t]{3}{*}{$A^{(b)}$} & 21.25 & \multirow{3}{*}{$\begin{array}{l}\text { Vialidad y } \\
\text { albañilería } \\
\text { variada }\end{array}$} & $\begin{array}{l}23.53 \\
(12.65)\end{array}$ & $72 \%$ & \multirow[t]{3}{*}{$78 \%$} \\
\hline & 36.17 & & $\begin{array}{l}30.41 \\
(19.53)\end{array}$ & $74 \%$ & \\
\hline & 90.42 & & $\begin{array}{l}23.23 \\
(12.35)\end{array}$ & $81 \%$ & \\
\hline
\end{tabular}

Fuente: elaboración propia a partir de los datos proporcionados por el Servicio de Prevención Ajeno de los casos y los informes de siniestralidad de las mutuas de AATT y EEPP. (a) años 2008 y 2009 respectivamente. (b) el caso A es en realidad una empresa con tres razones sociales, con dirección única, cuya razón social cambia cada cierto tiempo. (c) entre paréntesis la desviación respecto al sector. Año 2008

Las dos empresas con empleadores extranjeros están orientadas a la contratación de connacionales utilizando en la mayoría de los casos métodos informales de reclutamiento vinculados a la red social de su entorno, el empleador español contrata sobre todo trabajadores inmigrantes para los puestos de menos cualificación, o puertos de entrada, siendo el grupo más importante en número de la empresa.

A partir de los datos registrados de formación y siniestralidad laboral se analizan los efectos de las formaciones en las condiciones de trabajo, la aplicación de los conocimientos en los trabajos y la percepción de los trabajadores y empresarios del proceso formativo, combinando el análisis estadístico con el análisis cualitativo a través de la observación -de formaciones y trabajos en obra-, la entrevista y el estudio de los informes de los accidentes de trabajo (véase Hernández, 2012 pp. 161 a 211 y especialmente la etnografía en pp. 179 y ss.)

Cada técnica aporta información interesante para el objeto de estudio, que es utilizada para extraer un tipo de información concreta, como se aprecia en la siguiente tabla. 
Tabla 4. Técnicas de análisis

\begin{tabular}{cc}
\hline Registros de formación & $\begin{array}{c}\text { Contenido y duración de la forma- } \\
\text { ción. }\end{array}$ \\
Utilización de intérpretes. \\
Heterogeneidad del grupo.
\end{tabular}

Fuente: Elaboración propia a partir de datos del servicio de prevención ajeno y de los propios casos.

Se han visitado algunas obras importantes de la época: i. para el caso A, se han visitado numerosas obras de edificación, como la construcción de una biblioteca del Prat del Llobregat y también obras públicas como las realizadas en Casteldefells, las de reforma de la Plaça Lesseps de Barcelona o de la Plaça Europa de l'Hospitalet con numerosos trabajos de pavimentación y aceras; ii. para hacer un seguimiento del caso B, las obras de la Desalinizadora del Llobregat, donde se realizaron trabajos de albañilería diversos y de encofrado, las obras de reforma de la Gran Vía de Barcelona o las obras de mejora en la Estación de Ferrocarril de Sants, donde en todos los casos se realizaron diversos trabajos en cesión de trabajadores conocida como "administración” iii. en el caso C, se hizo el seguimiento en la construcción de un complejo de oficinas de Sant Cugat, donde se realizaron numeras actividades en la práctica de cesión de trabajadores o "administración” y el seguimiento en la construcción de un centro cívico en Santa Coloma de Gramanet.

Numerosas técnicas son utilizadas, entre ellas la participación en actividades con el grupo estudiado como las formaciones, como se describe en el siguiente fragmento de la exploración:

En enero y febrero de 2008 asisto a dos sesiones de formación, en una de ellas como formador. La lista de trabajadores muestra una considerable variedad de actividades y procedencias. (...) intento detectar a los que tienen más problemas para comunicarse y pregunto quién puede echar una mano traduciendo (...). Este tipo de 
estrategia me sirve también para comprobar la correspondencia entre un nombre extranjero y los problemas de comunicación. Fragmento de la etnografía del Caso A (Hernández, 2012).

Tabla 5. Resumen de datos de la investigación.

\begin{tabular}{cccc} 
Actividades & $\begin{array}{c}\text { Caso A empleador } \\
\text { español }\end{array}$ & $\begin{array}{c}\text { Caso B } \\
\text { empleador } \\
\text { extranjero }\end{array}$ & $\begin{array}{c}\text { Caso C } \\
\text { empleador } \\
\text { extranjero }\end{array}$ \\
\hline $\begin{array}{c}\text { Formaciones observa- } \\
\text { das/impartidas }\end{array}$ & 18 & 10 & 11 \\
\hline $\begin{array}{c}\text { Accidentes analizados } \\
\text { periodo 2007-20108 }\end{array}$ & 42 & 14 & 13 \\
\hline Visitas de obra & 17 & 13 & 3 \\
\hline $\begin{array}{c}\text { Entrevistas explorato- } \\
\text { rias a trabajadores }\end{array}$ & 5 & 5 & \\
\hline
\end{tabular}

Fuente: elaboración propia a partir de datos de la observación. (a) Se trata de accidentes para los que existe una investigación. Sus datos se cruzan con los datos de formación y siniestralidad del trabajador y de la empresa. (b) De los cuales 9 son extranjeros y 7 temporales. Para más datos véase la tabla 5 de la tesis doctoral (Hernández, 2012:167).

Además se realizan otras nueve entrevistas a encargados de empresas contratistas, representantes sindicales y empleadores del sector.

\section{Análisis crítico del modelo vigente}

A partir de nuestra interpretación de la redacción dada en el Convenio Colectivo de la Construcción, y adaptado a nuestro propio discurso, podemos distinguir entre 'formación específica de empresa' y 'formación general de sector', atendiendo al criterio de si se trata de formación transferible o no al mercado ${ }^{8}$.

El primer concepto es el más problemático. La 'formación específica’ se define por las necesidades del puesto de trabajo concreto $\mathrm{y}$, en sectores como la construcción, donde la producción se organiza en base a la subcontratación de los trabajos y una enorme flexibilidad organizativa, la adecuación y especificidad de los contenidos suponen un objetivo casi imposible de cumplir. Se pueden identificar numerosos obstáculos para ello, desde individuales como los referidos a la lengua de los trabajadores, a su nivel educativo reglado o a su experiencia previa en el sector, en la línea de análisis del capital humano, como los relacionados con la empresa y el sector, más en la línea de las teorías de las segmentación, como por ejemplo si se trata de un empleador intensivo que actúa como subcontratista, o si el empleador es extranjero y recluta al personal en su red social (como sucede en dos 
de los casos aquí analizados). También es importante tener en cuenta aspectos referidos a la organización del trabajo, como el nivel de descentralización de la producción, cosa que en el sector de la construcción suele ser muy habitual y donde la organización subcontratista deviene un elemento clave en la definición de las relaciones laborales y de los aspectos que influyen en la forma de organización e impartición de la formación ${ }^{9}$. De todo ello sirva de ejemplo el siguiente fragmento etnográfico:

Visitamos la Desalinizadora del LLobregat. Tenemos que dar un largo paseo para ver en qué se ocupan los 30 trabajadores de su empresa que se encuentran en la obra ese día. Hay variedad de tareas. Tres operarios están forrando columnas de hormigón para proteger la zona de tornillería de la base. Dos operarios son ecuatorianos y el otro marroquí. Otros dos operarios merodean por la zona. Todos están en “administración” (...). Hay otro trabajador que ayuda al topógrafo. Éste es un puesto muy deseado porque la carga de trabajo físico es mínima, pero se necesita a alguien que hable muy bien español. Encuentro otro grupo de trabajadores limpiando. Están lanzando escombros desde unos tablones colocados en ángulo sobre un contenedor al que acceden empujando la carretilla. Aprecio riesgo de caída. Otros seis trabajadores se ocupan en tareas diversas. Otros tres operarios realizan tareas de movimiento de tierras y también están aplanando el terrero. Uno de ellos se baja de un vehículo “minipala” y se sube a un rodillo aplanador. No espera a que baje el otro operario y sube mientras el otro se aparta. En lugar se sujetarse a la barra del chasis, se sujeta a los mandos del vehículo y provoca un movimiento brusco de la máquina. Etnografía caso B (Hernández, 2012)

De acuerdo con la legislación vigente en España, la formación 'específica’ debe proporcionarse en el trabajo a través de medios correctamente organizados y que puedan ser luego certificados por una entidad acreditada para ello. Su verdadero manual es la 'evaluación de riesgos' (obligatoria para todas las empresas y puestos de trabajo) y, por lo tanto, suele ser el Servicio de Prevención, ajeno o propio, (en adelante SPA o SPP) quien se ocupa de impartir periódicamente esta formación. Según la legislación, esta formación debe cumplir con una serie de requisitos de calidad referidos a la adecuación, suficiencia y especificidad, y además se exige que sea teórica y práctica.

El término referido a la "adecuación” puede ser interpretado en clave subjetiva, es decir, la formación debe ser la adecuada para los sujetos a los que se pretende formar y atender a sus necesidades en función de sus capacidades para seguir los conocimientos impartidos. Entre los elementos de la adecuación a los alumnos, no cabe duda, está la lengua en la que se imparte y el uso de intérpretes donde sea necesario. El convenio de la construcción se hace eco de tal problema cuando señala que:

(...) teniendo en cuenta la considerable presencia de los trabajadores inmigrantes en el sector, la formación e información dirigida a estos trabajadores será la adecuada y necesaria a sus características, especialmente en el caso de que desconozcan el idioma español.(Convenio Colectivo de la construcción, 2012; preámbulo). 
El análisis de los estudios de caso, muestra que muy pocas acciones formativas se han realizado con intérprete (ver tabla 6.).

\section{Tabla 6. Formación con intérprete y calidad de los medios}

\begin{tabular}{|c|c|c|c|c|c|}
\hline Caso & $\begin{array}{l}\text { Periodo de } \\
\text { análisis. }\end{array}$ & & $\begin{array}{l}\text { Trabajado- } \\
\text { res extran- } \\
\text { jeros for- } \\
\text { mados. }\end{array}$ & $\begin{array}{l}\text { trabajadores } \\
\text { formados con } \\
\text { interprete. }\end{array}$ & $\begin{array}{ll}\text { Calidad de la } & \text { de } \\
\text { interpretación. }\end{array}$ \\
\hline A & $\begin{array}{l}\text { Enero } 2008 \\
\text { diciembre } 2009\end{array}$ & $\mathrm{a}$ & 193 & $15 \%$ & $\begin{array}{l}\text { Poco intensa, } \\
\text { improvisada. Se } \\
\text { utiliza ejercicio con } \\
\text { foto y mesajes en } \\
\text { árabe. }\end{array}$ \\
\hline B & $\begin{array}{l}\text { Enero } 2008 \\
\text { enero } 2009\end{array}$ & $\mathrm{a}$ & 67 & $23 \%$ & $\begin{array}{l}\text { Improvisada por el } \\
\text { empleador. }\end{array}$ \\
\hline $\mathrm{C}$ & $\begin{array}{l}\text { Enero } 2008 \\
\text { diciembre } 2009\end{array}$ & & 31 & $9 \%$ & $\begin{array}{l}\text { Pobre calidad e } \\
\text { intensidad y sólo } \\
\text { para traducir pre- } \\
\text { guntas del ejercicio } \\
\text { final. }\end{array}$ \\
\hline
\end{tabular}

Fuente: observación y elaboración propia.

Siempre se han realizado de una manera muy improvisada, sin mucha traducción real, ya que se realizaron en un contexto poco favorecedor para ello por tratarse, a menudo, de grupos muy heterogéneos. Un porcentaje pequeño de la plantilla de trabajadores de las empresas estudiadas ha recibido algún tipo de ayuda para la comprensión de las formaciones, a pesar de que se trataba de empresas especializadas en la contratación de trabajadores inmigrantes y los trabajadores asistentes mostraban evidentes problemas de comprensión de la lengua española tal y como pudo observarse.

Meardi, Martín Artiles y Lozano (2013), en un trabajo comparativo de los mercados de trabajo de la construcción del Reino Unido y España destacan las diferencias importantes entre ambos países por lo que respecta a la formación en seguridad. Las empresas del Reino Unido tienen más experiencia adaptando la formación a las necesidades de los trabajadores que no hablan inglés, según se destaca en este trabajo, ya que desde 2004 la formación es impartida en diversas lenguas extranjeras sin necesidad de intérpretes

En nuestros estudios de caso (Hernández, 2012), muchos de los trabajadores entrevistados tenían problemas para expresarse con fluidez en castellano, incluso problemas de comprensión, pero aun así han pasado por varios cursos formativos impartidos únicamente en esta lengua. Como decía el siguiente testimonio: (...) a veces me pregunto cómo habrán formado a alguien que tu sabes que no se entera 
mucho. Lo veo un poco complicado en el caso de alguna persona. Entrevista a un encargado del caso A en obras de la Plaça Europa.

El término "suficiencia" se traduce habitualmente en tiempo dedicado a la formación, medido en minutos u horas, siendo lo habitual para una formación del SPA (o SPP) de dos horas, al menos sobre el papel. Aunque muchas de las formaciones observadas en las empresas estudiadas no han tenido tal duración sino menos.

Como la LPRL no establece una duración mínima, es la dinámica de las empresas del sector lo que ha influido en determinar la duración supuestamente suficiente, de tal manera que en el sector se ha llegado a convenir que con una formación de dos horas, repetida cada año, se puede cumplir suficientemente con las exigencias del artículo 19 de la LPRL. Así pues, la formación regulada en la LPRL suele ser conocida como "la formación de dos horas", porque esa ha sido su duración habitual y es así conocida por los empleadores para distinguirla de otras formaciones a las que también se llama por su duración. Así, a la formación de primer ciclo del Convenio se le conoce también como "la formación de las ocho horas".

El trabajo de campo de nuestra investigación ha permitido comprobar que la formación que se ha realizado en las diferentes obras rara vez ha llegado a las dos horas de duración. No obstante, la empresa contratista suele ser intransigente en esta cuestión y en ocasiones no da como bueno un certificado si no consta una duración de al menos dos horas. Se constata así que, para estas empresas, las apariencias son importantes, más incluso que el contenido de la formación.

Aunque en los tres casos analizados la formación ha sido planificada cada año, el momento de realizarla ha respondido a las necesidades del trabajo. Esta indefinición de calendario acaba por condicionar su contenido, pues se hace difícil mantener una secuencia coherente, y a menudo los temas tratados se repiten o se solapan con los impartidos en otras charlas. Por ello la formación es percibida por los trabajadores como muy repetitiva, pero no les importa porque priorizan el obtener el diploma.

La especificidad, respondería bastante acertadamente a la visión de Becker (1993) sobre tal formación. Una formación totalmente específica en prevención debe ser aquella que trata temas estrictamente de interés para los trabajadores ocupados en esa empresa y no en cualquier otra empresa, de manera que las situaciones de riesgos que se explican no son generales, sino realmente las experimentadas cotidianamente por los trabajadores empleados, y las medidas de prevención aquellas que, de común acuerdo con su empleador, se han establecido para la realización de sus trabajos.

La exploración efectuada en los casos de estudio ha mostrado que esta formación ha tendido a contener elementos generales:

Asisto a una formación del caso A. Hay trabajadores que han asistido a otras charlas anteriores. Se habla del casco y de las botas "otra vez" dicen algunos. No se habla del martillo neumático "eso si que interesa (...) a ti te quiero ver en esto" los 
extranjeros callan y no dicen nada, parece que no entienden mucho. Fragmento de la etnografía caso A.

Este tipo de charlas de corta duración y contenido generalista permiten justificar el cumplimiento formal de los requisitos exigidos en la LPRL, pero las enseñanzas impartidas, sólo teóricas, tienen un efecto limitado sobre la forma de trabajar y la sobre productividad, lejos de la tesis del capital humano.

Las empresas estudiadas en la presente investigación ejercen tareas de subcontratistas para una empresa central. La contratista utiliza aquellos servicios como herramientas de flexibilidad externa, externalizando costes laborales, una parte de la producción, o servicios concretos a la producción, fundamentalmente tareas de peonaje, sencillas, duras físicamente y de duración indeterminada y no continuada, que vienen a resolverse de manera más ágil con ayuda de estos subcontratistas intensivos.

El reclutamiento y gestión de personal en estas empresas subcontratistas es bastante complejo, y depende de las demandas de contratación, la reducción de equipos o el desplazamiento de trabajadores de una obra a otra. Estos tres elementos determinan las necesidades de flexibilidad interna de los subcontratistas intensivos. Los casos estudiados afrontan lo que se ha denominado "problemas de ajuste entre producción y empleo” (Martín Artiles, 1992) sin poder evitar que ello afecte en cierto sentido a la seguridad en el empleo.

La formación cumple una función preventiva cuando sirve al propósito de proporcionar a los trabajadores el conocimiento preventivo adecuado para evitar los accidentes, reducir los riesgos y mejorar las condiciones de trabajo, ya sea antes de que suceda un daño o después de un accidente o suceso no deseado. No disponer de la formación adecuada es considerado como un factor de riesgo en sí mismo.

Para la empresa la organización de cursos de prevención supone una inversión en tiempo y dinero menor que la formación en habilidades productivas, que requiere más tiempo o una dilatada experiencia en la propia empresa. Los efectos sobre la productividad de esta última actividad se dan por supuesto, mientras que en general se pone en duda la utilidad de los cursos de prevención, como se aprecia en los siguientes testimonios:

La formación es un trámite que hay que pasar lo más rápidamente posible y en el menor coste, no me fastidies. Si quieres dame los papeles, si hay confianza. Yo ya sé que tu eres muy serio no voy a pensar mal de ti. Comentario del empleador en una visita a obras de Sant Cugat. Caso C. Fragmento de la etnografía (Hernández, 2012).

Si la formación está muy bien, pero que les vas a decir (...) el trabajo tiene que salir y luego ellos hacen lo que quieren (...) a mi me "apretan” éstos para acabar a tiempo y qué, no me vengas con tonterías de arnés o no sé qué, (...) Entrevista a empleador marroquí del caso B. (ibídem.)

Aun así, la utilidad de la formación en prevención de riesgos laborales es más evidente en ciertos casos: si en el momento de recibir una Inspección de trabajo, un 
trabajador no ha realizado la formación exigida, esto conllevará una sanción que reducirá los beneficios que se obtengan por ese trabajo y aumentará los costes por hora de trabajo de ese trabajador. Disponiendo de la credencial la empresa puede evitar una sanción económica.

Algunos trabajos de investigación han resaltado algunos efectos positivos de la formación preventiva sobre el trabajo, como la reducción de las interrupciones, la mejora en la sensación de seguridad percibida por los trabajadores, la reducción de la rotación, la pérdida o caída de herramientas o el aumento de la calidad en el trabajo (Goldenhar et. al. 2001). De ahí que la formación preventiva, formal o informal, es vista por los prevencionistas por su función en la reducción de los accidentes y sobre la producción.

En los casos analizados, la exigencia legal de disponer de trabajadores formados en prevención de riesgos implica una carrera por disponer del documento que diga que se ha hecho esta formación, dando cumplimiento formal y documental a la ley. Pero es obvio que una prevención adecuada exigiría algo más, ya que un papel no sirve para evitar un accidente o para proteger las vías respiratorias de un trabajador. La preocupación por el rol credencialista de la formación viene de lejos. Ruiz Frutos (1999) decía hace más de una década:

debemos plantearnos si no estaremos más preocupados en que los trabajadores dispongan de un certificado acreditativo de su formación sin valorar su aprendizaje real. El número de trabajadores formados o las horas recibidas son indicadores que difícilmente valoran la utilidad de dicha formación (op.cit. pp 170-171).

Los datos empíricos obtenidos en esta investigación confirman que la formación en prevención de riesgos laborales ha servido a un propósito económico-productivo más que a un objetivo estrictamente preventivo, pues no existen muchos estímulos para mejorarla o anticiparse a los requerimientos del mercado. La empresa hace la formación cuando está "acorralada” por las circunstancias, como se muestra en el siguiente fragmento de la etnografía:

si no se hace la formación mañana no podemos entrar a trabajar el lunes. Tienes que organizar un curso para estos siete como sea o pierdo dinero. Escucho este comentario del empelador del caso A. Siempre es lo mismo, cuando lo necesitan. Fragmento de la etnografía del caso A (Hernández, 2012).

Los certificados de prevención han sido utilizados en todos los casos para poder entrar a los centros de trabajo, cobrar facturas pendientes o evitar sanciones de la Inspección de Trabajo. En esta línea podemos decir que la formación es considerada como una inversión necesaria que da sus frutos a corto plazo.

Entre los diversos tipos de formación preventiva, la que supone un coste mayor para el empleador es la formación exigida por el Convenio del sector de la construcción. Se observa que las empresas procuran participar en la financiación de esta formación lo menos posible, ya que las cualificaciones y competencias adquiridas mediante esta formación son transferibles de una empresa a otra. De 
acuerdo con la teoría del capital humano, una formación perfectamente general sería igualmente útil en muchas firmas.

En algunos casos el coste total del proceso es asumido por la empresa. En nuestros estudios de caso, tal cosa sucede cuando se trata de un empleador étnico, por el peso que los lazos étnicos parecen tener en el reclutamiento de connacionales. En este caso, la red social supera las explicaciones propias del capital humano, pues los beneficios obtenidos con tal reclutamiento son otros (rapidez, dominio sobre el reclutado o dependencia éste, nivel de entrega en el trabajo, posibilidades de flexibilidad, etc.). Se observa que en otras ocasiones, este tipo de formación es asumido por las empresas mediante arreglos parciales: la formación se realiza los sábados (fuera de horario laboral) en un centro subvencionado, pagando las horas como trabajadas para algunos trabajadores, y para otros imponiendo la formación como una condición para continuar en la empresa. Y en otros casos, se realiza un sábado pagándoles la empresa el curso, pero sin pagarles las horas de asistencia como trabajadas.

De acuerdo con Becker (1993), si el trabajador abandona esta empresa para trabajar en otra, la formación general puede dar rendimientos a las empresas que no la han impartido. Este es el caso de la formación del Convenio de la construcción, ya que otorga una credencial que el trabajador puede hacer valer ante cualquier empresa del sector. Cuando se trata de trabajadores extranjeros empleados por un connacional, las posibilidades de que esa credencial sea utilizada casi en exclusiva por el empleador que la ha patrocinado son elevadas, debido a la dependencia de los trabajadores con su red social, empezando por el empleador.

Por lo que respecta a la formación del SPA (o SPP), su papel es el de una formación específica del puesto de trabajo y, por tanto, no válida en ninguna otra empresa. La relevancia de esta formación quedó parcialmente ensombrecida cuando las contratistas empezaron a exigir el cumplimiento del Convenio colectivo, que conllevaba una formación más genérica.

En el caso de esta formación específica, la empresa no tiene más remedio que asumir su coste, pues a ello obliga la legislación. Pero la práctica observada en los casos estudiados es la de demorarla lo más posible o evitar la realización hasta que sea imprescindible, entre otras cosas, porque su cliente, la contratista, tampoco lo considera imprescindible y, desde el punto de vista del empleador, hacer parar a los trabajadores para darles esta formación supone perder horas de trabajo. No obstante, a veces, un cliente puede condicionar el cumplimiento de un subcontrato o acuerdo a la realización de esta formación específica, no tanto con fines preventivos sino más bien formales, para evitar responsabilidades.

Por lo que respecta a la calidad de esta formación, en casos así se han observado déficits que afectan a la transferencia y al proceso de asimilación de conocimientos: no hay formación práctica, rara vez se han utilizado intérpretes ni material traducido, y a veces tiene una duración menor de lo pactado. En el caso de la formación del Convenio además hay que añadir los incumplimientos con el programa del curso de formación, ya que muchas veces no se respetan o no se terminan. 
Estos hechos aglutinan muchos de los elementos que nos permiten una lectura credencialista del proceso de formación, tales como la influencia de la tipología de empresa, que al estar en condiciones de inferioridad en la cadena de subcontratación ve mermada su capacidad de organización, los incumplimientos del programa de formación establecido o no tener en cuenta la lengua en la que se expresan los trabajadores.

En términos de la Teoría del capital humano, cualquier formación preventiva que sea exigida por un cliente, aunque sea deficiente, será una buena inversión, pues dará sus frutos a corto plazo y el empleo de los trabajadores formados depende de ello. En los casos analizados, la formación se ve como una inversión, pero no en términos de ahorro de costes por accidentes de trabajo o bajas laborales, sino como un medio para que una unidad de trabajo, con un valor previsto en horas de servicio, pueda ser utilizada en la producción. Un trabajador no formado no puede producir.

La transmisión de conocimientos útiles al trabajador no puede medirse con exactitud, sobre todo en un sector como la construcción, pero sí puede observarse en combinación con el tratamiento estadístico de los accidentes, método que se considera válido por otros estudios precedentes como el de Goldenhar et al (2001) o Bena et al (2009) y que es utilizado también en este estudio de casos en el que se inspira este escrito (Hernández, 2012).

El análisis de los accidentes "pre” y "post-training” permite dirigir la mirada hacia la hipótesis que la formación preventiva está lejos de lograr su objetivo (véase la tabla 7). No se aprecian grandes diferencias en la duración de las bajas en los tres casos de estudio. Parece que la condición "ser inmigrante" y "ser temporal" es más determinante pues ubica al trabajador en tareas con más riesgos.

Subyace una falta de satisfacción de las necesidades de los trabajadores, tanto previa a los daños como posteriores a ellos, que refuerza la tesis credencialista. Por ejemplo, en el caso A el 78\% de los accidentados por sobreesfuerzo tenía formación específica. Se trata de 25 de los 67 accidentes registrados en el periodo 2008-2009, de los cuales, 7 se deben al uso del martillo neumático. En las visitas se ha observado la dureza de dicho trabajo y de las diversas tareas de manipulación de peso. En ninguna formación de las presenciadas se practica el uso de este equipo ni el manejo de cargas.

Tabla 7. Principales indicadores años 2008-9

\begin{tabular}{|c|c|c|c|c|}
\hline \multicolumn{2}{|c|}{ Indicador } & Caso A & Caso B & Caso C \\
\hline \multicolumn{2}{|c|}{$\begin{array}{l}\text { \% accidentados } \\
\text { temporales }\end{array}$} & $61,2 \%$ & $77,3 \%$ & $46,7 \%$ \\
\hline \multicolumn{2}{|c|}{$\begin{array}{l}\% \text { accidentados con } \\
\text { formación }\end{array}$} & $73,8 \%$ & $50 \%$ & $80 \%$ \\
\hline $\begin{array}{l}\text { Principal } \\
\text { duración } \\
\text { accidentados } \\
\text { formación. }\end{array}$ & $\begin{array}{r}\text { rango } \\
\text { bajas } \\
\text { con }\end{array}$ & 1 a 5 días $(36,2 \%)$ & 6 a 15 días $(58,3 \%)$ & 1 a 5 días (41,7\%) \\
\hline Principal & rango & 6 a 15 días $(40 \%)$ & 1 a 5 días $(72,7 \%)$ & 1 a 5 días (50\%) \\
\hline
\end{tabular}




\begin{tabular}{|c|c|c|c|c|}
\hline $\begin{array}{l}\text { duración } \\
\text { accidentados } \\
\text { formación. } \\
\end{array}$ & $\begin{array}{r}\text { bajas } \\
\text { sin }\end{array}$ & & & \\
\hline $\begin{array}{l}\% \text { tempo } \\
\text { accidentados } \\
\text { formación. }\end{array}$ & $\begin{array}{r}\text { alidad } \\
\text { sin }\end{array}$ & $94,1 \%$ & $91,7 \%$ & $66,7 \%$ \\
\hline $\begin{array}{l}\text { \% tempo } \\
\text { accidentados } \\
\text { formación. }\end{array}$ & $\begin{array}{r}\text { alidad } \\
\text { con }\end{array}$ & $47,9 \%$ & $60 \%$ & $41,7 \%$ \\
\hline $\begin{array}{l}\text { Principal } \\
\text { accidentados } \\
\text { formación } \\
\end{array}$ & $\begin{array}{r}\text { causa } \\
\text { con }\end{array}$ & Golpes (41,7\%) & Golpes $(66,7)$ & Golpes (80\%) \\
\hline $\begin{array}{l}\text { Principal } \\
\text { accidentados } \\
\text { formación. }\end{array}$ & $\begin{array}{r}\text { causa } \\
\text { sin }\end{array}$ & $\begin{array}{l}\text { Golpes (35,3\%) pero } \\
\text { todo lo musculoes- } \\
\text { quelético (postura y } \\
\text { sobreesfuerzo) suman } \\
41,1, \% \text {. }\end{array}$ & Golpes (90\%). & $\begin{array}{l}\text { Golpes, postura } \\
\text { inadecuada y otros } \\
\text { en igual propor- } \\
\text { ción. }\end{array}$ \\
\hline
\end{tabular}

Fuente: elaboración propia a partir de datos registrados.

Además, también se ha detectado una falta de interés por la realización de formaciones correctoras tras un accidente ya que una vez se tiene un diploma y mientras sea válido, parece poco eficiente realizar otra acción formativa. En los casos analizados, después de un accidente no se realiza una formación correctora. En los tres casos estudiados y a lo largo de una periodo de 5 años sólo se recoje una actividad de este tipo después de un accidente.

Las entrevistas realizadas permiten observar también que los mecanismos más elementales de prevención, como el estado de alerta, la percepción de los riesgos o la prudencia, son adquiridos por los trabajadores más a través de la propia experiencia en la obra que con la formación en el aula. Como diría Collins (1989), el aprendizaje de profesiones específicas descansa a menudo más en la experiencia en el propio trabajo que en la enseñanza formal. Este aprendizaje, puede y debe incluir, también una parte del conocimiento preventivo necesario para hacer el trabajo. Los trabajadores con limitaciones de idioma son un ejemplo aún más evidente de la importancia del proceso de ósmosis en la adquisición de una cultura preventiva en el lugar de trabajo.

Otro aspecto que corrobora la visión credencialista de la formación preventiva es el salario. Como se ha podido apreciar en el trabajo de campo, el sueldo de los trabajadores no experimenta cambio alguno con la adquisición de la credencial. Esto se debe a que la obligación de disponer de la formación preventiva es transversal a todas las categorías profesionales. Todos los grupos, desde peón hasta capataz, deben tener la misma formación.

Algunas diferencias entre trabajadores son apreciables debido al dominio del idioma. Ambas aspectos, el idioma y la experiencia en el puesto son más importantes en la definición del salario, especialmente en el caso del empleador 
español. ${ }^{10}$ Trabajadores con años en la empresa, sin un dominio avanzado del idioma, pueden tener más dificultades para completar su proceso de aprendizaje en el trabajo, por lo que un peón puede permanecer en esa categoría varios años. Estos trabajadores son condenados a tareas sencillas y similares durante todo el tiempo de estancia en la empresa. Sin embargo, todos ellos tendrán la misma formación preventiva, tanto la específica del SPA (o SPP) como la formación general del Convenio.

Por lo tanto, dados los escasos efectos de la formación preventiva sobre el salario, la teoría del capital humano no acaba de explicar satisfactoriamente la situación, puesto que tener una formación preventiva, específica o general, no mejora las expectativas salariales del trabajador.

\section{Conclusión}

La formación en prevención de riesgos laborales es un elemento clave para la seguridad y salud en el trabajo, y así lo reconoce la principal legislación que regula esta cuestión (la LPRL 31/1995). El desarrollo de esta normativa puso en práctica una serie de actividades formativas de carácter específico (adecuadas a cada puesto de trabajo concreto, en función de los riesgos allí existentes) tradicionalmente impartidos por los técnicos de los Servicios de Prevención (ajenos o propios). Adicionalmente, el IV Convenio de la construcción 2007-2011 reguló unos cursos obligatorios en base a dos ciclos, uno inicial de 8 horas de duración y otro de especialización de 20 horas de duración denominado segundo ciclo de oficios o puestos de trabajo.

Los cursos impartidos por los Servicios de Prevención tienen un valor escaso en el mercado de trabajo ya que la credencial que producen es un certificado de realización de la actividad que sólo pueden ser utilizado en un puesto de trabajo de una empresa concreta (donde se imparte la formación). En cambio, la credencial dispensada con la formación del Convenio tiene efectos sobre el mercado de trabajo, pues el trabajador es el poseedor de dicho diploma y puede utilizarlo en cualquier otra empresa. Estas circunstancias han influido poderosamente en el tipo de formación que se ha venido llevando a cabo en el sector de la construcción.

Los efectos de estas formaciones sobre la prevención de riesgos laborales en los tres subcontratistas observados son escasos, ya que las verdaderas necesidades de formación no suelen ser satisfechas. Por un lado porque no hay una correspondencia entre las actividades que se realizan y el contenido de la formación. Y por otro lado, porque dada la importancia de la mano de obra extranjera en el sector, las necesidades idiomáticas no suelen ser tenidas en cuenta y raramente se intenta adaptar la formación a las capacidades de comprensión de los trabajadores.

En el fondo, esta situación refleja que los incentivos para hacer la formación son principalmente de tipo credencial, pues el empleador sólo pretende con el acto formativo superar una barrera que haga del trabajador una unidad productiva utilizable en el mercado de trabajo. La respuesta a la pregunta que se formulaba en 
el título de este artículo la encontramos aquí: la formación sirve para superar barreras formales impuestas por el mercado de trabajo y las regulaciones pero en general no alcanza a satisfacer sus verdaderos objetivos preventivos.

No obstante, creemos que es necesario dialogar con las teorías del capital humano y el credencialismo, pues ambas aportan elementos explicativos de interés. La teoría del capital humano tiene una cierta capacidad explicativa sobre el desarrollo de las estrategias de los individuos: la competencia por los escasos recursos de empleo, la exigencia cada vez mayor de saber hacer y el valor de uso de las titulaciones confieren una cierta importancia a la interpretación desde la teoría del capital humano. El diploma ofrece un signo inequívoco al mercado de trabajo, una señal, de que esa persona ha hecho algo de mérito.

Pero inmediatamente también hay que subrayar la capacidad explicativa de la teoría del credencialismo: los títulos tienen también un valor de cambio en el mercado de trabajo. El credencialismo nos ofrece una interpretación diferente de las titulaciones: la adquisición de títulos no siempre está relacionada con el conocimiento y el saber hacer.

El punto de partida de este debate no tiene por qué llevar a la contraposición de enfoques teóricos, sino a su complementariedad. Por ejemplo, ambas teorías reconocen un valor a la formación en el puesto de trabajo, y en especial a la formación informal a la que se atribuye ciertos efectos en la productividad, desde una visión funcionalista, y en la adquisición de conocimientos mediante la práctica, desde una visión neoweberiana. Es además necesario ver en el credencialismo, no sólo un diagnóstico de la formación preventiva, sino también una expresión del fracaso en la consecución de los objetivos funcionales de la formación como medio para mejorar la productividad y reducir la siniestralidad.

A la vista de estos resultados se justifica pensar en otros modelos de formación más basados en la práctica y más centrados en las necesidades de los trabajadores. Es necesario valorar más la formación aquí llamada específica de empresa y exigirla con criterio, en base un análisis de necesidades que haga diana en las carencias reales de los trabajadores. En este sentido, sería necesario un desarrollo reglamentario del artículo 19 de la ley de prevención sobre el contenido práctico de la formación y los recursos necesarios para satisfacer las necesidades específicas detectadas. Los convenios colectivos, como el de la construcción, deben desarrollar mecanismos de evaluación del conocimiento práctico-preventivo que se considere mínimo para trabajar en el sector.

No obstante es conveniente aclarar, que los resultados no pueden extrapolarse a empresas demasiado diferentes a las estudidadas, por más que estas representan una parte importante de la organización de la producción en el sector. Es necesario un estudio más amplio cuyos resultados puedan extrapolarse al conjunto de sector. 


\section{Notas finales}

1.- Se destaca también en las conclusiones finales de la IV edición de los Encuentros Nacionales de Educación y Formación en Prevención de Riesgos Laborales que se celebraron en Andalucía en 2011.

2.- La competencia es definida por la Ley 5/2002 de las Cualificaciones y de la Formación Profesional como el conjunto de conocimientos y capacidades que permiten el ejercicio de la actividad profesional conforme a las exigencias de la producción y del empleo. Aunque no sea formación profesional del sistema educativo, comparte los propósitos del RD 1147/2011 y debe inspirarse en los objetivos fijados por la Unión Europea que recogen la necesidad de incrementar el nivel de formación y cualificación tanto de los jóvenes en edad escolar como de la población trabajadora.

3.- Entrevista a representante sindical especializado en formación. La postura de los sindicatos motivó una nota aclaratoria de la inspección de trabajo y seguridad social para matizar el alcance de la postura sindical.

4.- Estas horas pueden ser convalidadas con la realización de un primer curso de 20 horas o con la realización del curso de técnico de nivel básico de 60 horas de duración.

5.- Según se oriente hacia la consecución de unos fines laborales determinados, la formación puede agruparse en los grupos de formación en el empleo o de formación para el empleo. La formación en el empleo o formación en el trabajo es un elemento útil para conservar el trabajo o mejorar en él porque es la vía para adaptarse a las nuevas técnicas productivas y/o a las nuevas condiciones de trabajo y organización y evitar que los conocimientos queden obsoletos. La formación para el empleo es un mecanismo de acceso al mercado de trabajo. La formación y la cualificación se han convertido en las claves para acceder al empleo y mantenerse en él (Martín Artiles y Lope, A, 1999).

6.- Los criterios de la Inspección de Trabajo y Seguridad y Social y del Departament de Treball de la Generalitat de Catalunya, sobre como debe ser una formación específica que complemente a la formación general se puede leer en el apartado de preguntas mas frecuentes en el siguiente sitio web: http://www20.gencat.cat/portal/site/empresaiocupacio/menuitem.dd2cfc37bb1b69c 0a6740d63b0c0e1a0/?vgnextoid=fcb37e7ee95bf210VgnVCM1000008d0c1e0aRC RD\&vgnextchannel=fcb37e7ee95bf210VgnVCM1000008d0c1e0aRCRD\&vgnextf $\mathrm{mt}=$ default\&newLang=es_ES (última consulta junio 2013)

7.- En la fase de exploración se recogieron algunos comentarios de trabajadores y empresarios acerca de los inconvenientes sobre la realización de la actividad y también sobre su utilidad. No obstante el trabajo de Foment del Treball Nacional (2007) recoge una visión positiva entre los trabajadores encuestados. En el trabajo de Goldenhar et al (2001) se identifican algunas barreras a la formación.

8.- Para estos autores "la formación profesional general la formación profesional específicas suelen ir unidas. Además la mayoría de los planes de formación contienen elementos de ambos tipos". Pese a ello "la Teoría del Capital 
Humano diferencia entre estos dos modalidades de formación (Becker op. cit) y considera que unos puestos de trabajo se distinguen de otros en función del grado en que exigen de las personas que los ocupan cualificaciones y conocimientos transferibles de unas empresas a otras” (Alcaide, González y Flores, 1996).

9.- A pesar de que en el convenio colectivo de la construcción se refiere en su preámbulo a la formación regulada como específica, nuestro análisis quiere mostrar que no es tan específica como debería ser, siguiendo un criterio preventivo, ni tampoco en atención a un criterio sociológico. En este trabajo se pretende destacar la importancia de la especificidad no solo por la particular interpretación que se hace de este concepto referenciado en el artículo 19 de la ley de prevención, sino por su destacada importancia como elemento preventivo.

10.- En esta misma forma de análsis en la que convergen ambas visiones, la del capital humano y la de las teoría de la segmentación se mueve el estudio de Martín Artiles, Lopez-Roldán y Oscar Molina (2011) que analiza la movilidad ascendente vertical de los trabajadores inmigrantes en España a partir de la Muestra Continua de Vidas Laborales (MCVL).

11.- En el empleador español es más difícil progresar a puestos mejor retribuidos sin hacer progresos con el idioma, aunque las habilidades siguen siendo importantes, relativamente, en trabajos de peonaje inespecífico, con mucha movilidad funcional de una tarea física sencilla a otra.

\section{Bibliografía}

Alcaide Castro, M. González Rendon, M. y Flores Saborido I. (1996) Mercado de trabajo, reclutamiento y formación en España. Madrid, Pirámide.

Becker, G. (1993). Human Capital: A Theoretical and Empirical Analysis with Special Reference to Education. The University of Chicago Press. Third Edition. Chicago.

Becker, P y Morawetz, J. (2004). Impact of health and Safety Education: comparison of workers Activities Before and After Training. American Journal of Industrial Medicine. Vol. 46, 63-70.

Bena, A., et al (2009), "Effectiveness of the Training Program for Workers at Construction Sites of the High-Speed Railway Line Between Torino and Novara: Impact on Injury Rates”. American Journal of Industrial Medicine. Vol. 52, 965-972.

Bourdieu, P. y Passeron, L.C. (1969), Los estudiantes y la cultura. Barcelona. Nueva colección labor, Núm. 49. 
Bowles, S. y Gintis, H. (1985). La instrucción escolar en la América capitalista: la reforma educativa y las contradicciones de la vida económica. Madrid. Siglo XXI de España.

Collins, R. (1989), La Sociedad Credencialista: sociología histórica de la educación y la estratificación. Torrejón de Ardoz. Akal.

Encuentros Nacionales de Formación y Educación en prevención de riesgos laborales. IV edición. Universidad Internacional de Andalucía. Junio de 2011.

Foment del Treball Nacional (2007). La incidencia de la inmigración en el sector de la construcción en materia de prevención de riesgos laborales. Estudio de investigación. Barcelona. Foment del Treball Nacional,

Goldenhar, L. Stacey, K. y Colligan, M. (2001) Health and Safety Training in a Sample of open-shop construction companies. Journal of Safety Research. Vol. 32, 237-252.

Hernández Salguero, D. (2012) Formación Preventiva e inmigración en el sector de la construcción. Tesis Doctoral. Departament de Sociologia, Universitat Autònoma de Barcelona.

Martin Artiles, A y Lope, A. (1999), "Sirve la formación para el empleo”. Papers. Vol. 58, 39-73.

Martin Artiles, A, Lopez- Roldan, P. y Molina, O. (2011), "Movilidad ascendente de la inmigración en España: ¿̇asimilación o segmentación ocupacional?” Papers. Vol. 96 Núm. 4, 1335-1362.

Meardi, G., Martín Artiles, A. y Lozano Riera, M. (2013), “constructing uncertainty: unions and migrant labour in construction in UK and Spain". Journal of Industrial Relations. Vol. 1 Núm. 55, 174-189.

Ruíz Frutos, C. (1999), “Formación en prevención de riesgos laborales” Cuadernos de Relaciones Laborales. Núm.14, 163-172. 